\title{
EDITORIAL
}

\section{Predicting VBAC Success: From Theory to Clinical Practice}

To facilitate evidence-based decision-making, decision aids are often used as adjunct tools for patient counseling. A recent example is a web-based tool that provides patient-specific estimates of childhood neurological outcome following periviable preterm birth. ${ }^{1}$ In this issue of the journal, Bill Grobman and colleagues describe and compare two statistical models that could serve as useful decision aids for potential vaginal birth after cesarean (VBAC) candidates. ${ }^{2}$

Based on data from 9616 women attempting VBAC across 19 medical centers, they evaluated factors associated with VBAC success and created two predictive models. They compared two nomograms: one based on factors "available early in pregnancy," and one based on factors only available "immediately prior to VBAC attempt." The predictive model based on factors available in early pregnancy includes maternal age, body mass index, race, and prior obstetrical outcomes. ${ }^{3}$ The model based on factors available immediately prior to VBAC attempt incorporate additional obstetric factors such as labor induction, preeclampsia, and cervical status. Although the purpose of the study was to compare the predictive abilities of these two models, it is compelling to demonstrate how the data from these nomograms could be translated into improving clinical care.

Unless a woman with a prior cesarean delivery has a clear contraindication to $\mathrm{VBAC}$, most providers have an initial discussion regarding VBAC. The timing of this discussion is typically around mid-pregnancy. Most often in routine practice women are provided with a "ball-park" estimate rather than a patient-specific estimate. For example, many clinicians will discuss an overall VBAC success rate between 60 and $80 \%$ and then tell a candidate she is probably at the upper or lower end of the spectrum based on an estimate of her individual risk factors.

Using the first nomogram (available at http:// www.bsc.gwu.edu/mfmu/vagbirth.html), a provider can input the relevant factors and receive a probability of VBAC success (with 95\% confidence intervals). For some women with a very low probability of success, a decision to forgo a VBAC attempt and schedule repeat cesarean delivery might be determined at this time. In a prior analysis, Grobman et al demonstrated that women with a low probability of VBAC success had higher rates of maternal and neonatal morbidity. ${ }^{4}$ Thus, a probability of success $<50 \%$ may be a reasonable threshold to set to select out women for repeat cesarean delivery (rather than VBAC attempt) based on their low chance for success and higher risk for complications. For women who have a higher probability for success (e.g., $>70 \%$ ) and are interested in $\mathrm{VBAC}$, a tentative plan for $\mathrm{VBAC}$ attempt could be made based on the same risk/benefit assessment. Routine care would continue until spontaneous labor or the need for iatrogenic delivery. If a woman remains a VBAC candidate, the second nomogram could be used to provide a more updated probability of success that would incorporate additional obstetric factors. If this updated probability is low (e.g., $<50 \%$ ), then she and her provider may reconsider and proceed toward repeat cesarean delivery.

Given the predictive abilities of both models, there is strong rationale to use either nomogram for patient counseling depending on the time of assessment. Providing a patient-specific probability of VBAC success which is evidence-based will no doubt improve the quality of care delivered to women considering VBAC. Whether or not providing this patient-specific data would actually translate into a change in VBAC attempt rates is unknown. It is plausible that the use of these two nomograms in tandem could improve VBAC success rates and decrease morbidities associated with a failed attempt. Whether selection of optimal candidates based on their pre-test probability of VBAC success improves outcomes requires testing in a clinical trial.

$$
\text { Sean C. Blackwell, M.D. }{ }^{1}
$$

\section{REFERENCES}

1. Tyson JE, Parikh NA, Langer J, Green C, Higgins RD; National Institute of Child Health and Human Development Neonatal Research Network. Intensive care for extreme
${ }^{1}$ Director, Larry C. Gilstrap M.D. Center for Perinatal and Women's Health Research, Department of Obstetrics, Gynecology, and Reproductive Sciences, University of Texas Health Science Center at Houston.

Address for correspondence and reprint requests: Sean C. Blackwell, M.D., Department of Obstetrics, Gynecology, and Reproductive Sciences, University of Texas Health Science Center at
Houston, 6410 Fannin Street, Houston, TX 77030 (e-mail: Sean. Blackwell@uth.tmc.edu).

Am J Perinatol 2009;26:691-692. Copyright (C) 2009 by Thieme Medical Publishers, Inc., 333 Seventh Avenue, New York, NY 10001, USA. Tel: +1(212) 584-4662.

DOI 10.1055/s-0029-1241060. ISSN 0735-1631. 
prematurity-moving beyond gestational age. N Engl J Med 2008;358(16):1672-1681

2. Grobman WA, Lai Y, Landon MB, et al; National Institute of Child Health and Human Development (NICHD) MaternalFetal Medicine Units Network (MFMU). Does information available at admission for delivery improve prediction of vaginal birth after cesarean? Am J Perinatol 2009;26(10):693-702

3. Grobman WA, Lai Y, Landon MB, et al; National Institute of Child Health and Human Development (NICHD) Maternal-
Fetal Medicine Units Network (MFMU). Development of a nomogram for prediction of vaginal birth after cesarean delivery. Obstet Gynecol 2007;109(4):806-812

4. Grobman WA, Lai Y, Landon MB, et al; Eunice Kennedy Shriver National Institute of Child Health and Human Development Maternal-Fetal Medicine Units Network. Can a prediction model for vaginal birth after cesarean also predict the probability of morbidity related to a trial of labor? Am J Obstet Gynecol 2009;200:e1-e6 